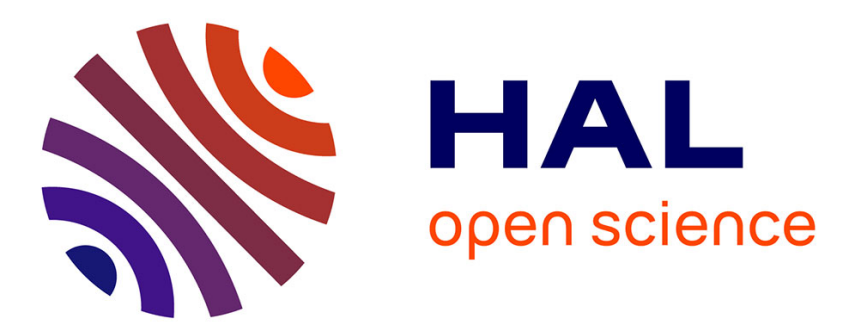

\title{
Origine des oscillations ioniques dans une source d'ions à confinement magnétique et à extraction axiale
}

\author{
J. Machet
}

\section{To cite this version:}

J. Machet. Origine des oscillations ioniques dans une source d'ions à confinement magnétique et à extraction axiale. Revue de Physique Appliquée, 1975, 10 (6), pp.453-461. 10.1051/rphysap:01975001006045300 . jpa-00243945

\section{HAL Id: jpa-00243945 https://hal.science/jpa-00243945}

Submitted on 1 Jan 1975

HAL is a multi-disciplinary open access archive for the deposit and dissemination of scientific research documents, whether they are published or not. The documents may come from teaching and research institutions in France or abroad, or from public or private research centers.
L'archive ouverte pluridisciplinaire HAL, est destinée au dépôt et à la diffusion de documents scientifiques de niveau recherche, publiés ou non, émanant des établissements d'enseignement et de recherche français ou étrangers, des laboratoires publics ou privés. 
Classification

Physics Abstracts

0.673

\title{
ORIGINE DES OSCILLATIONS IONIQUES DANS UNE SOURCE D'IONS A CONFINEMENT MAGNÉTIQUE ET A EXTRACTION AXIALE
}

\author{
J. MACHET \\ Laboratoire de Physique Ionique, U. E. R. des Sciences \\ 123, rue A.-Thomas, 87100 Limoges, France \\ (Reçu le 20 février 1975, révisé le 13 juin 1975, accepté le 16 juin 1975)
}

\begin{abstract}
Résumé. - Dans le présent article nous présentons la réalisation et les caractéristiques d'une source d'ions destinée à l'étude fondamentale de certains phénomènes physiques intervenant dans les sources à champ magnétique et à extraction axiale.

Nous avons mis en évidence des oscillations de type ionique de fréquence de l'ordre du $\mathrm{MHz}$. Ces oscillations sont excitées par le mouvement individuel des ions dans le puits de potentiel radial créé par le faisceau d'électrons rapides. L'apparition et la disparition de ces oscillations, liées à des variations importantes de la répartition du potentiel de charge d'espace à l'intérieur de la chambre, entraînent des discontinuités dans le courant ionique extrait.
\end{abstract}

Abstract, - In the present article we describe the building and the caracteristics of an ion source used in the basic research into certain physics phenomena found in magnetically confined ion sources with axial extraction.

We have emphasised ion oscillations having a frequency of the order of $\mathrm{MHz}$. These oscillations are caused by individual ion movements in the radial potential field created by the accelerated electron beam. The appearance and disappearance of these oscillations, linked with important variation in the distribution of the potential inside the chamber, lead to a certain discontinuity in the current at the outlet.

1. Introduction. - L'utilisation croissante des ions dans de nombreux domaines de la recherche fondamentale ou appliquée entraîne le développement et la construction d'un grand nombre de sources d'ions. Or ce développement se heurte à de nombreuses difficultés. Les phénomènes mis en jeu, en particulier ceux qui prennent naissance dans le milieu ionisé d'où sont extraits les ions sont complexes et encore souvent mal connus.

Dans le travail qui suit, après avoir décrit la source que nous avons mise au point, nous montrerons le rôle joué par des instabilités de type ionique.

2. Dispositif expérimental. - 2.1 PRINCIPE. - Les ions sont produits par bombardement d'une cible gazeuse par un faisceau d'électrons rapides.

Le montage (Fig. 1) comprend trois parties principales :

- le canon à électrons ;

- la chambre d'ionisation avec le dispositif de confinement et d'extraction des ions ;

- le dispositif analyseur.
Dans l'élaboration de ce montage nous avons voulu, avant tout, que les paramètres physiques, commandant le fonctionnement de la source, soient accessibles à la mesure directe avec une bonne précision et soient indépendants les uns des autres.

2.2 CANON A ÉLECTRONS. - C'est un canon à focalisation de Pierce classique. La pervéance est égale à $2 \times 10^{-6} \mathrm{~A} \cdot \mathrm{V}^{-3 / 2}$. Afin de pouvoir faire varier l'énergie des électrons sans modifier l'intensité du courant émis, nous avons ajouté une deuxième anode. Cette énergie peut varier de 400 à $4000 \mathrm{eV}$. L'intensité totale du courant est comprise entre quelques $\mathrm{mA}$ et $100 \mathrm{~mA}$. L'utilisation d'une cathode à oxydes de préférence à une cathode en tungstène a permis de réduire la puissance de chauffage de 200 à 18 W. Cette cathode est en nickel pur recouvert d'un mélange actif dont on trouvera la composition dans la référence [1]. Cette diminution de la puissance de chauffage permet d'atteindre rapidement l'équilibre thermique et de rendre le dégazage pratiquement négligeable. Les qualités optiques du canon ne sont pas modifiées. Le fonctionnement 


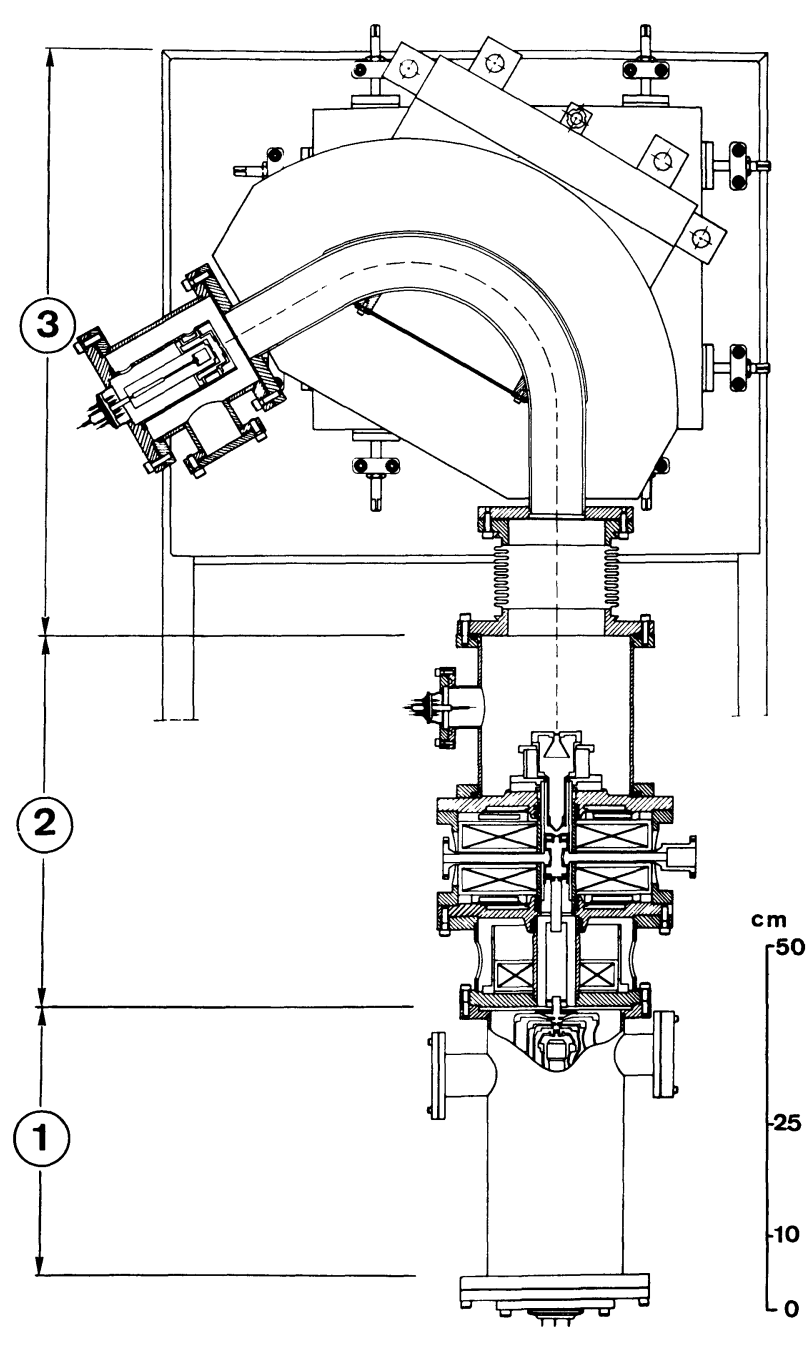

Fig. 1. - Vue d'ensemble du montage : 1. Canon à électrons ; 2. Dispositif de confinement et d'extraction des ions ; 3. Analyseur.

n'est pas altéré par les remises à l'air libre. Les cathodes ainsi mises au point ont des durées de vie supérieures à $1000 \mathrm{~h}$.

Afin que le fonctionnement du canon à électrons ne soit pas perturbé par le champ magnétique intense régnant au niveau de la chambre d'ionisation, nous avons cherché à éloigner au maximum le canon de cette dernière. Le diaphragme d'entrée de la chambre $\mathrm{O}_{1}$ est situé à $18 \mathrm{~cm}$ de la seconde anode $\mathrm{A}_{2}$ du canon à électrons. Pour que les électrons puissent pénétrer dans le champ magnétique intense régnant dans la chambre, nous avonc cherché à réduire la divergence du faisceau en plaçant après le canon à électrons une lentille magnétique $b$. Un simple réglage de l'intensité du courant circulant dans la lentille, en fonction des paramètres du faisceau électronique, permet aux électrons de pénétrer dans la chambre. Le coefficient de transmission est variable suivant les conditions expérimentales. Pour un diaphragme d'entrée $\mathrm{O}_{1}$ de diamètre $1,5 \mathrm{~mm}$ et pour un faisceau d'intensité égale à $10 \mathrm{~mA}$ l'énergie des électrons valant $800 \mathrm{eV}$, le rapport de l'intensité du courant électronique pénétrant dans la chambre à l'intensité du courant émis par le canon est supérieur à $85 \%$.
2.3 Chambre D'IONiSATION, DISPOSITIF DE CONFINEMENT ET D'EXTRACTION DES IONS. - Elle est constituée par une enceinte cylindrique dont l'axe coïncide avec celui du faisceau électronique ionisant.

Un champ magnétique, de révolution autour de l'axe de la chambre, créé par les bobines $B_{1}$ et $B_{2}$ (Fig. 2), confine le faisceau électronique et le milieu ionisé dans un volume aussi restreint que possible. Suivant le sens du courant circulant dans les bobines nous pouvons obtenir une forme fuseau des lignes de champ ou bien une forme cuspidée. L'induction magnétique sur l'axe peut varier de 0 à $0,16 \mathrm{~T}$.

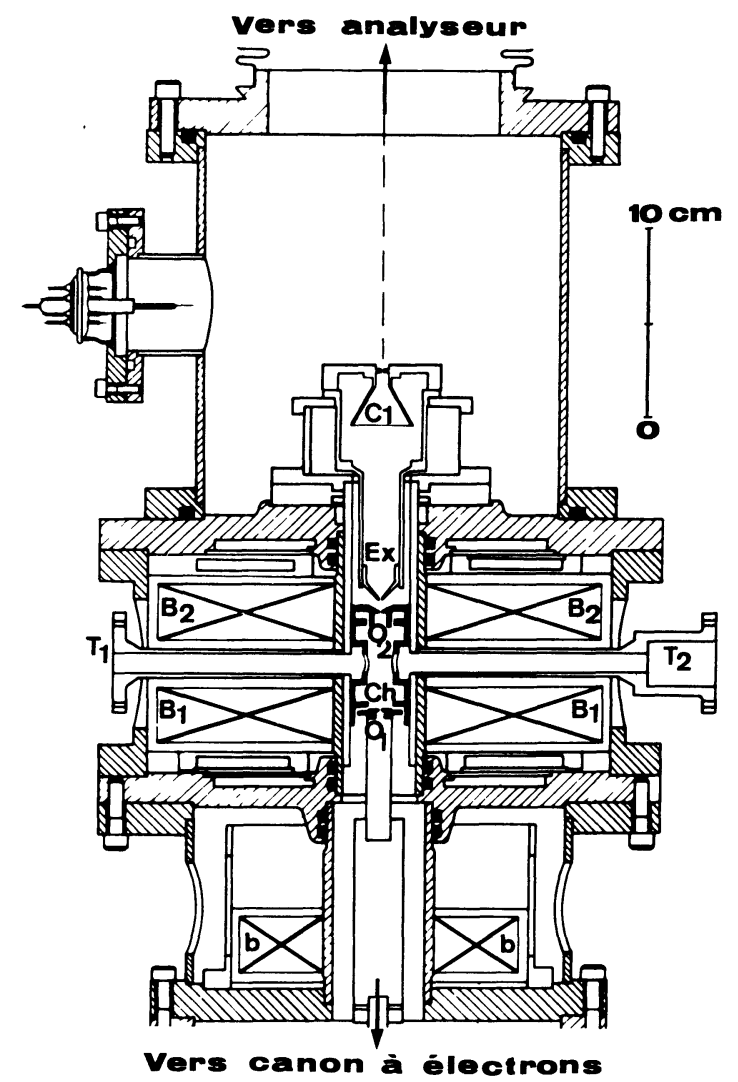

FIG. 2. - Dispositif de confinement et d'extraction des ions.

Le gaz est introduit à travers une fuite de débit réglable par l'intermédiaire de la tubulure radiale $T_{1}$. La pression $P_{\mathrm{s}}$ dans la chambre, qui peut varier de $2 \times 10^{-6}$ à $1 \times 10^{-3}$ torr, est mesurée à l'aide d'une jauge à ionisation reliée à la tubulure radiale $T_{2}$. Une mesure indirecte de la pression source $P_{\mathrm{s}}$ par une méthode de débit [2] nous a permis de contrôler que les indications de la jauge étaient correctes. La précision des mesures est supérieure à $5 \%$.

Les ions sont extraits axialement à l'aide de l'électrode $\mathrm{E}_{\mathbf{x}}$. La tension d'extraction, mesurée par rapport à la chambre peut varier de -800 à $-5000 \mathrm{~V}$. Cette tension est toujours supérieure à la différence de potentiel sous laquelle sont accélérés les électrons rapides. Ces derniers se réfléchissent dans le domaine d'extraction et retraversent la chambre. L'intensité du faisceau électronique ionisant, $I^{-}$, sera donc égale au double de 
l'intensité du faisceau électronique pénétrant dans la chambre par le diaphragme $\mathrm{O}_{1}$.

Les ions extraits sont récupérés sur le collecteur $C_{1}$. Un orifice de faible diamètre percé dans le fond de ce collecteur permet d'analyser les ions créés sans perturber sensiblement la valeur de l'intensité du courant recueilli sur $\mathrm{C}_{1}$.

2.4 Dispositif ANAlYSEUR. - Il est constitué par un électro-aimant à indice stigmatique dont le pouvoir de résolution est égal à 300 . Les ions analysés sont recueillis sur un cylindre de Faraday. Une électrode polarisée négativement par rapport à ce cylindre évite les erreurs dues à l'émission d'électrons secondaires. Enfin un diaphragme percé d'un trou dont le diamètre est égal à celui de l'image de l'orifice de sortie des ions de la chambre définit la partie utile du faisceau.

3. Etude expérimentale : mise en évidence de discontinuités dans le courant ionique extrait. - Le libre parcours des électrons étant grand devant les dimensions de la chambre et en admettant qu'il n'y a pas d'effets secondaires l'intensité du courant ionique extrait $I_{\text {ex }}^{+}$peut se mettre sous la forme :

$$
I_{\mathrm{ex}}^{+}=R_{\mathrm{s}} \sigma I^{-} n_{0} l
$$

- $\sigma:$ section efficace totale d'ionisation;

- $I^{-}$: intensité totale du faisceau électronique ionisant ;

- $n_{0}$ : nombre de neutres par unité de volume, $n_{0}$ est proportionnel à la pression $P_{\mathrm{s}}$ régnant dans la chambre ;

- $l$ : longueur d'une trajectoire aller des électrons dans la chambre. La détermination numérique des trajectoires montre que $l$ est supérieure d'environ $10 \%$ à la longueur de la chambre;

- $R_{\mathrm{s}}$ : rendement d'extraction de la source, il dépend en particulier de la tension d'extraction des ions, du champ magnétique régnant dans la chambre, des dimensions géométriques du diaphragme $\mathrm{O}_{2}$, de la charge d'espace.

Afin de simplifier nous ne rappellerons que les résultats expérimentaux concernant l'influence de la pression et de l'intensité du faisceau électronique ionisant sur l'intensité du courant ionique extrait. Les conclusions que l'on peut en tirer peuvent d'ailleurs s'étendre à l'étude de l'influence des autres paramètres.

\subsection{INFLUENCE DE LA PRESSION SUR L'INTENSITÉ DU} COURANT IONIQUE EXTRAIT $I_{\mathrm{ex}}^{+}$. $-I_{\mathrm{ex}}^{+}$représente la somme des courants ioniques reçus par le collecteur $\mathrm{C}_{1}$ et l'électrode d'extraction $\mathrm{E}_{\mathrm{x}}$. $I_{\mathrm{ex}}^{+}$varie comme l'intensité du courant ionique recueilli par le collecteur $\mathrm{C}_{1}$, ce dernier étant polarisé négativement par rapport à l'électrode $E_{x}$ pour éviter l'erreur due aux électrons secondaires. $I_{\mathrm{ex}}^{+}$est supérieur d'environ $10 \%$ au courant recueilli par $C_{1}$. Ceci est dû au courant ionique recueilli par $\mathrm{E}_{\mathrm{x}}$. Dans ces conditions il nous est apparu logique d'étudier directement le courant $I_{\mathrm{ex}}^{+}$, ce qui nous permettait de simplifier le montage électrique. En outre nous pouvons considérer que même avec ce montage relativement simple l'erreur due aux électrons secondaires est sans doute faible du fait de la présence du champ magnétique intense régnant au niveau de l'électrode d'extraction et de la position du collecteur $C_{1}$ qui est pratiquement entouré par l'électrode $E_{x}$.

La figure 3 reproduit l'influence de la pression sur le courant ionique extrait.

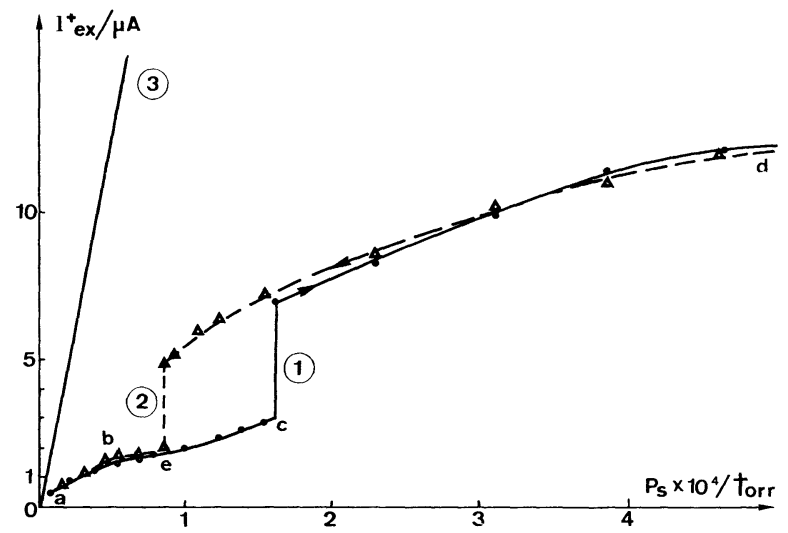

FIG. 3. - Influence de la pression sur le courant ionique extrait $I^{-}=12,4 \mathrm{~mA}, V^{-}=800 \mathrm{~V}, V_{\mathrm{ex}}^{+}=4000 \mathrm{~V}$, argon).

La courbe 1 représente la variation de $I_{\mathrm{ex}}^{+}$lorsque l'on fait croître la pression. Dans tout le domaine (a c) le courant ionique est pratiquement constant, puis pour une valeur critique de la pression, il croît brusquement, sa valeur est multipliée par 2 ou 3 suivant les cas. Ensuite le courant ionique extrait croît d'une façon monotone. Lorsque l'on fait décroître la pression la courbe représentative du courant ionique extrait (courbe 2) a même allure que la précédente. L'intensité du courant ionique extrait décroît brutalement pour une valeur critique de la pression inférieure à celle correspondant à la discontinuité précédente. La courbe 3 représente le courant ionique qui serait extrait si le coefficient $R_{\mathrm{s}}$ de la relation (3.1) était égal à 1, c'est-àdire qu'elle représente la variation du courant que l'on pourrait recueillir si tous les ions créés étaient extraits.

3.2 INFLUENCE DE L'INTENSITÉ DU FAISCEAU ÉLECTRONIQUE IONISANT $I^{-}$SUR L'INTENSITÉ DU COURANT IONIQUE EXTRAIT $I_{\mathrm{ex}}^{+}-I^{-}$représente l'intensité totale du faisceau électronique ionisant. Elle est égale à la somme des intensités des faisceaux incident et réfléchi traversant la chambre. L'intensité du faisceau incident est mesurée en déterminant le courant recueilli sur l'électrode d'extraction des ions et le collecteur $\mathrm{C}_{1}$, cette électrode et $\mathrm{C}_{1}$ étant reliés au même potentiel que la chambre. Quand nous appliquons une tension d'extraction des ions, supérieure à la tension d'accélération des électrons, ces derniers sont réfléchis dans le domaine d'extraction des ions et retraversent la chambre. 
L'intensité du courant recueilli sur le diaphragme de sortie des ions représente moins de $1 \%$ du courant électronique total. Dans ces conditions nous pouvons admettre que :

$$
I^{-}=2 I_{\mathrm{ex}}^{-}
$$

où $I_{\mathrm{ex}}^{-}$représente l'intensité du courant électronique incident.

La figure 4 représente l'influence de $I^{-}$sur l'intensité du courant ionique extrait.

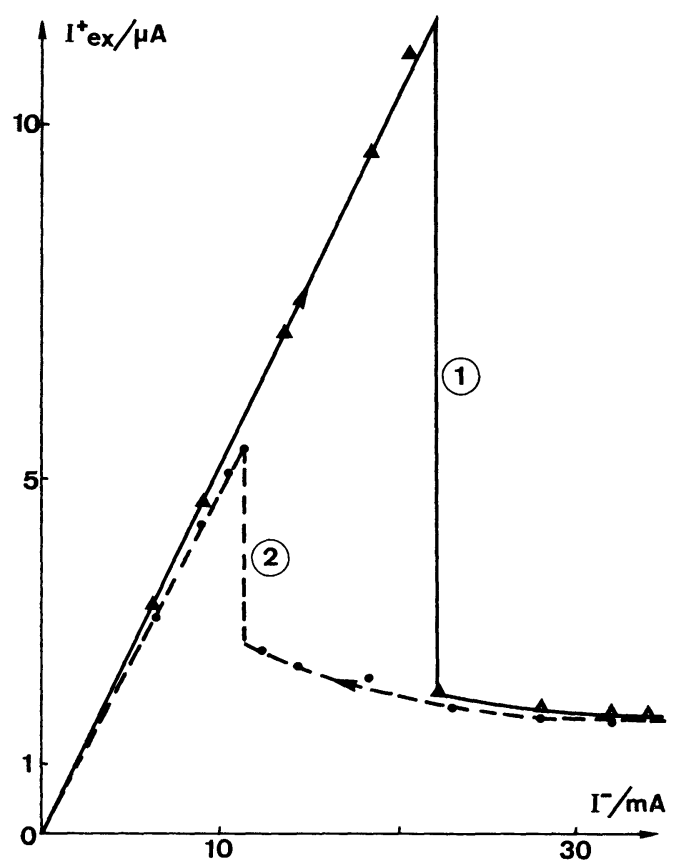

Fig. 4. - Influence de l'intensité du courant électronique ionisant sur l'intensité du courant ionique extrait

(argon, $P_{\mathrm{s}}=1,8 \times 10^{-4}$ torr, $V^{-}=800 \mathrm{~V}, V_{\mathrm{ex}}^{+}=4000 \mathrm{~V}$ ).

Lorsque l'on fait croitre $I^{-}, I_{\mathrm{ex}}^{+}$croît proportionnellement à $I^{-}$, puis pour une valeur critique de $I^{-}$il décroît brusquement, ensuite il est sensiblement constant quel que soit $I^{-}$(courbe 1). Si nous faisons décroître $I^{-}$nous obtenons une courbe qui a sensiblement même allure que la précédente. Le courant ionique est pratiquement constant jusqu'à une valeur critique de $I^{-}$où il croît brusquement puis décroît proportionnellement à $I^{-}$(courbe 2).

Ce qui frappe le plus sur les différentes courbes obtenues ce sont les brusques discontinuités qui apparaissent dans le courant ionique extrait. Ces discontinuités peuvent s'expliquer de 2 manières différentes:

- soit par un changement brutal du régime d'ionisation, la relation (3.1) n'étant plus applicable ;

- soit par une modification des conditions d'extraction des ions.

L'expérience, en particulier la mesure du courant ionique global créé, nous a montré que la première hypothèse est peu vraisemblable.
Les discontinuités sont dues à une modification des conditions d'extraction provoquée par l'apparition ou la disparition d'oscillations de fréquence voisine du $\mathrm{MHz}$. Leur apparition provoque en général une brusque décroissance du courant ionique extrait (discontinuités des courbes 2 et 1 des Fig. 3 et 4). Leur disparition entraîne son accroissement (discontinuités des courbes 1 et 2 des Fig. 3 et 4). Ces oscillations entraînent une modulation des courants recueillis sur les diverses électrodes. Les photos 1 et 2 de la figure 5 représentent respectivement l'oscillogramme et le spectre de fréquence de ces oscillations recueillies sur le tube de glissement reliant le canon à électrons à la chambre d'ionisation.

En outre les diverses discontinuités sont accompagnées d'une brusque variation de l'énergie des ions liée sans doute à une modification du potentiel de charge d'espace au point où est créé l'ion. Nous allons étudier successivement ces deux résultats.

4. Etude des oscillations. - L'étude expérimentale de ces oscillations montre que la fréquence fondamentale dépend des paramètres du faisceau électronique ionisant (intensité $I^{-}$et énergie $\mathrm{eV}^{-}$des électrons rapides) et de la masse atomique du gaz à étudier.

La figure 6 montre que la fréquence est proportionnelle à la racine carrée de l'intensité $I^{-}$du faisceau électronique ionisant. Les différentes droites correspondent aux résultats obtenus avec les gaz rares et l'azote. La figure 7 montre que la fréquence est inversement proportionnelle à la racine carrée de la masse atomique ou molaire de ces mêmes gaz.

La proportionnalité de la fréquence à la racine carrée de l'intensité du faisceau électronique ionisant et à l'inverse de la racine carrée de la masse atomique nous a amené à penser que ce sont des oscillations ioniques et plus particulièrement des oscillations transversales de type plasma comme le suggère Hernquist [3].

4.1 MOdèle Des oscillations DES IONS. - Tout d'abord il faut remarquer que, compte tenu de la symétrie de révolution apparente du système, il semblerait logique de considérer un mouvement collectif radial des ions. Cependant ce modèle, que nous avons envisagé en premier, conduit à une valeur théorique de la fréquence supérieure à la valeur mesurée expérimentalement [4]. Nous avons alors été amené à considérer un mouvement transversal, provoqué certainement par une dissymétrie du faisceau électronique, le faisceau réfléchi ne coïncidant pas parfaitement avec le faisceau incident. Pour interpréter ce mouvement considérons en première approximation que le faisceau d'électrons rapides puisse être assimilé à un cylindre de rayon moyen, $a$. Nous pouvons admettre que les trajectoires des électrons rapides ne sont pas modifiées par le mouvement des ions. Supposons qu'à un instant donné, $t$, les ions soient contenus dans le cylindre défini par le faisceau d'électrons rapides. Si tous les ions sont 


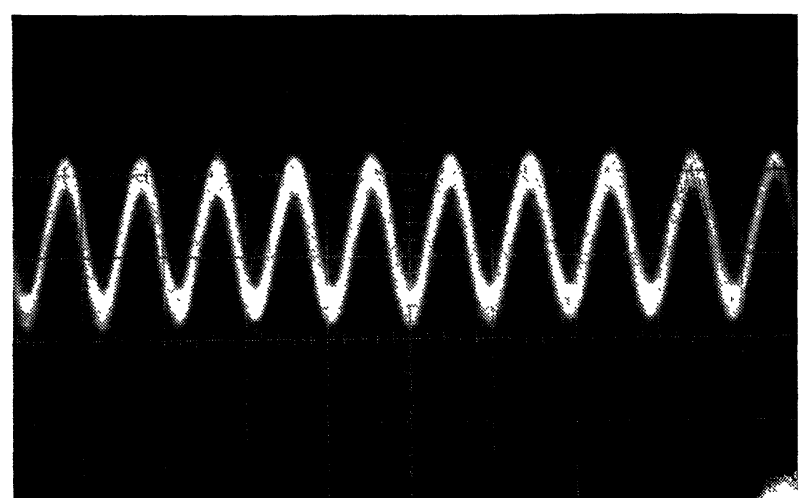

Рното 1. - Oscillogramme des oscillations (1 $\mu \mathrm{s} /$ division; $1 \mathrm{mV} /$ division).

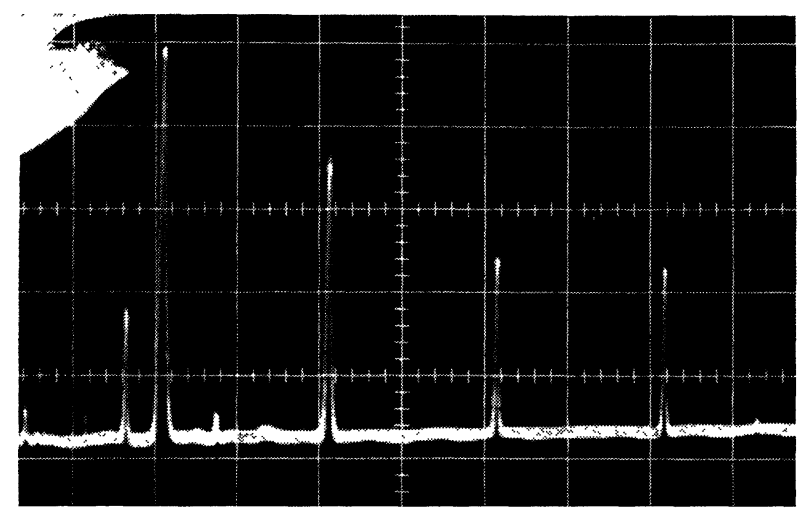

Рното 2. - Spectre de fréquence des oscillations $(0,5 \mathrm{MHz} /$ division, fréquence centrale : $2,5 \mathrm{MHz} ; 10 \mathrm{db} /$ division origine $-80 \mathrm{db})$.

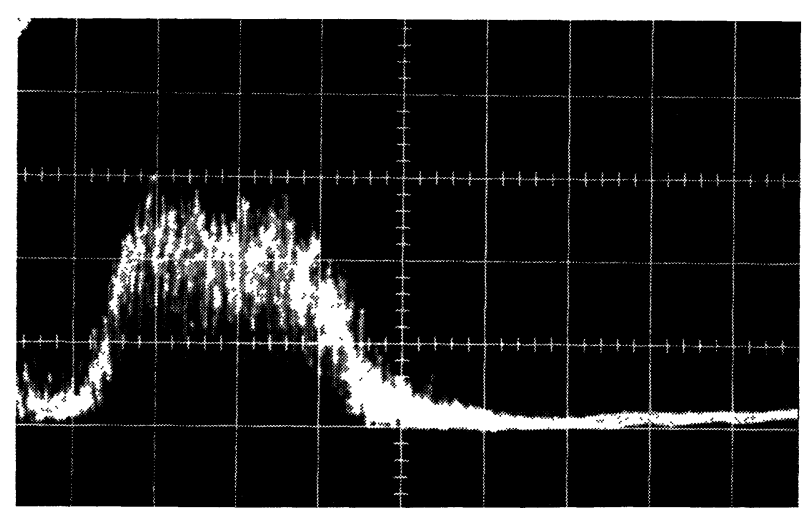

Рното 3. - Analyse du bruit de fond se produisant aux très basses pressions avant l'apparition de l'oscillation cohérente : $(0,5 \mathrm{MHz} /$ division, fréquence centrale : $2,5 \mathrm{MHz} ; 10 \mathrm{db} / \mathrm{cm}$ origine- $80 \mathrm{db}$ ).

Fig. 5. - Etude à l'oscillographe et à l'analyseur de spectre des oscillations.

animés du même mouvement, à l'instant $t+\mathrm{d} t$, ils seront contenus dans un cylindre dont l'axe aura subi un déplacement transversal $\xi$ par rapport à celui du cylindre défini par le faisceau d'électrons rapides comme l'indique la figure 8 .

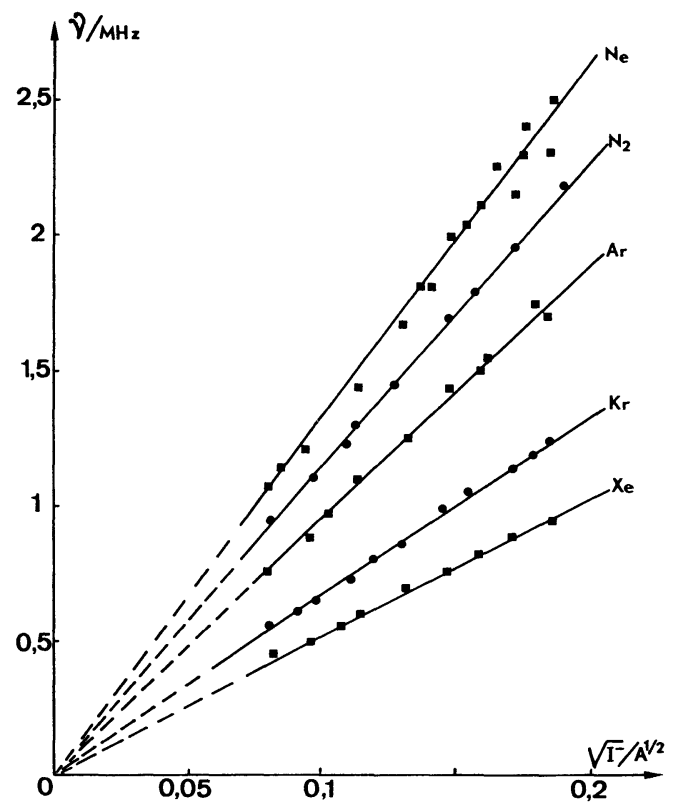

Fig. 6. - Influence de l'intensité du faisceau électronique ionisant sur la fréquence des oscillations $\left(V^{-}=800 \mathrm{~V}\right.$, $\left.V_{\mathrm{ex}}^{+}=4000 \mathrm{~V}\right)$.

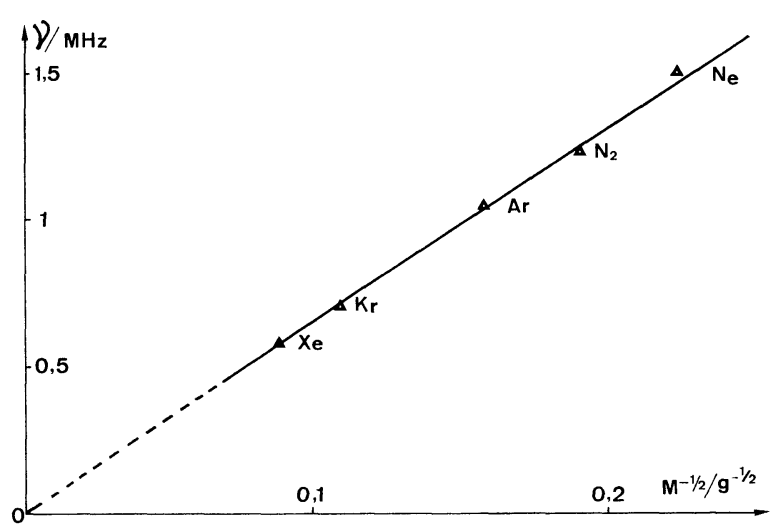

Fig. 7. - Influence de la masse atomique sur la fréquence des oscillations $\left(I^{-}=12,4 \mathrm{~mA}, V^{-}=800 \mathrm{~V}, V_{\mathrm{ex}}^{+}=4000 \mathrm{~V}\right)$.
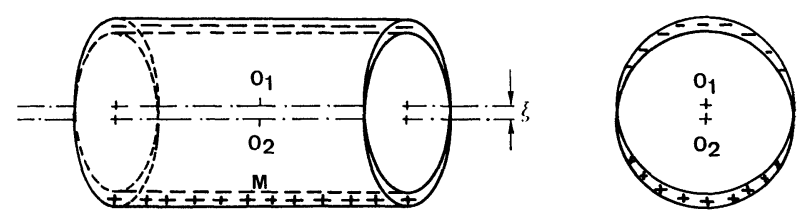

Fig. 8. - Interprétation de l'oscillation transversale des ions.

L'application du théorème de Gauss montre que le champ créé en un point $M$ par cette nouvelle répartition est :

$$
\mathbf{E}=\frac{1}{2 \varepsilon_{0}}\left(\rho^{-} \mathbf{O}_{1} \mathbf{M}+\rho^{+} \mathbf{O}_{2} \mathbf{M}\right)
$$

$\rho^{-}$: densité de charges des électrons rapides ;

$\rho^{+}:$densité de charges due aux ions. 
Si nous supposons :

$$
\begin{aligned}
\rho^{+} & =-\rho^{-}, \\
\mathbf{E} & =\frac{\rho^{+}}{2 \varepsilon_{0}} \mathbf{O}_{2} \mathbf{O}_{1} .
\end{aligned}
$$

Le champ est uniforme à l'intérieur de la répartition de charges. Les ions sont alors soumis à une force de rappel proportionnelle au déplacement, ils vont osciller autour de leur position d'équilibre avec une fréquence :

$$
v=\frac{1}{2 \pi}\left(\frac{q \rho^{+}}{2 m^{+} \varepsilon_{0}}\right)^{1 / 2} .
$$

En tenant compte de la relation (3.2) et de l'expression de $\rho^{-}$en fonction de l'intensité $I^{-}$du faisceau d'électrons rapides, de sa section $S$ et de la vitesse $v^{-}$ des électrons :

$$
\rho^{-}=-\frac{I^{-}}{S v^{-}}
$$

nous pouvons exprimer la fréquence en fonction des paramètres macroscopiques.

Dans le système M. K. S. A. nous obtenons :

$$
v=4,82 \times 10^{5}\left(\frac{I^{-}}{S M \sqrt{V^{-}}}\right)^{1 / 2}
$$

$M$ est la masse atomique du gaz étudié et $V^{-}$la tension d'accélération des électrons rapides.

Remarque. - L'hypothèse $\rho^{+}=-\rho^{-}$que nous venons de faire se trouve confirmée par l'expérience. En effet, il n'est pas possible de mettre en évidence ces oscillations pour les très basses pressions (région $\mathrm{a}-\mathrm{b}$ des courbes de la Fig. 3). Dans ce cas on n'observe qu'un bruit de fond incohérent comme l'indique la photo 3 de la figure 5 .

4.2 VÉRIFICATION EXPÉRIMENTALE DU MODÈLE PROPOSÉ. - La relation (3.3) qui montre la proportionnalité de la fréquence à la racine carrée de l'intensité et à l'inverse de la racine carrée de la masse atomique se trouve vérifiée par les graphes des figures 6 et 7 .

Par contre la fréquence n'est pas proportionnelle à l'inverse de la racine quatrième de la tension d'accélération des électrons rapides. Ceci peut sans doute se justifier facilement de la manière suivante : lorsque nous faisons varier $I^{-}$ou $M$ les autres paramètres de la relation 3.3 ne sont pratiquement pas modifiés, alors que toute variation de $V^{-}$entraîne celle de la section $S$ du faisceau d'électrons rapides, le rayon des trajectoires dépendant de $V^{-}$.

En outre ce rayon dépend de la valeur de l'induction magnétique sur l'axe. Or comme le laisse supposer la disposition des bobines $B_{1}$ et $B_{2}$ (Fig. 2), la valeur de l'induction magnétique au niveau des diaphragmes d'entrée, $\mathrm{O}_{1}$, et de sortie, $\mathrm{O}_{2}$, est supérieure à sa valeur au centre de la chambre. Nous avons été amené à définir un rayon moyen, $a$, tel que :

$$
a=\frac{a_{1}+a_{2}}{2}
$$

où $a_{1}$ et $a_{2}$ sont respectivement les rayons du faisceau au niveau du diaphragme d'entrée et au centre de la chambre.

Le rayon des trajectoires croît avec la tension d'accélération $V^{-}$des électrons rapides. Cependant le rayon moyen du faisceau dans la chambre va tendre vers une limite car $a_{1}$, rayon du faisceau pénétrant par le diaphragme $O_{1}$, ne pourra jamais être supérieur au rayon de ce diaphragme.

Nous avons pris pour $a$ une expression de la forme :

$$
a=a_{0}\left(1-\mathrm{e}^{-c V^{-}}\right)
$$

$a_{0}$ est obtenu en faisant :

$$
a_{1}=a_{0_{1}}
$$

$c$, est calculé en considérant que pour $V^{-}=1100 \mathrm{~V}$, nous faisons une erreur inférieure à $5 \%$ en admettant que le rayon du faisceau $a_{1}$ est égal à celui du diaphragme d'entrée $a_{01}$. Nous avons pris $V^{-}=1100 \mathrm{~V}$ car à partir de cette valeur, nous sommes obligés d'augmenter l'intensité totale du courant électronique émis par le canon pour maintenir l'intensité $I^{-}$constante à l'intérieur de la chambre lorsque l'on augmente $V^{-}$.

Dans ces conditions, pour un diaphragme d'entrée de $0,75 \mathrm{~mm}$ de rayon :

$$
a_{0}=1 \mathrm{~mm} \text {. }
$$

Nous aurions peut-être pu trouver une loi limite plus simple pour $a$. Nous avons tout simplement raisonné par analogie avec de nombreuses relations limites trouvées en physique. L'accord entre les résultats expérimentaux et la relation théorique que nous allons mettre en évidence nous a amené à considérer cette hypothèse comme plausible.

En remplaçant $S$ par sa valeur, l'expression de la fréquence prend alors la forme suivante dans le système M. K. S. A. :

$$
\begin{aligned}
v=2,72 \times 10^{8}\left(\frac{I^{-}}{M}\right)^{1 / 2} \times & \times \frac{1}{\left[1-\exp \left(-2,6 \times 10^{-3} V^{-}\right)\right] \sqrt[4]{V^{-}}} .
\end{aligned}
$$

Sur les figures 6 et 7 les points correspondent aux valeurs expérimentales, les droites en trait plein représentent les variations de $v$ en fonction de $\left(I^{-}\right)^{1 / 2}$ ou de $(M)^{-1 / 2}$ données par la relation (3.4). Donc non seulement la fréquence varie proportionnellement à $\left(I^{-}\right)^{1 / 2}$ et $(M)^{-1 / 2}$ mais est aussi en bon accord avec les valeurs prévues par la relation (3.4).

La figure 9 représente la variation de la fréquence en fonction de la tension d'accélération des électrons 
rapides. Dans tous les cas $I^{-}$est constant et égal à 12,4 mA. Les valeurs expérimentales sont représentées par des points. Les courbes en trait plein représentent la variation de la fonction (3.4) dans laquelle $M$ et $I^{-}$ sont supposés constants.

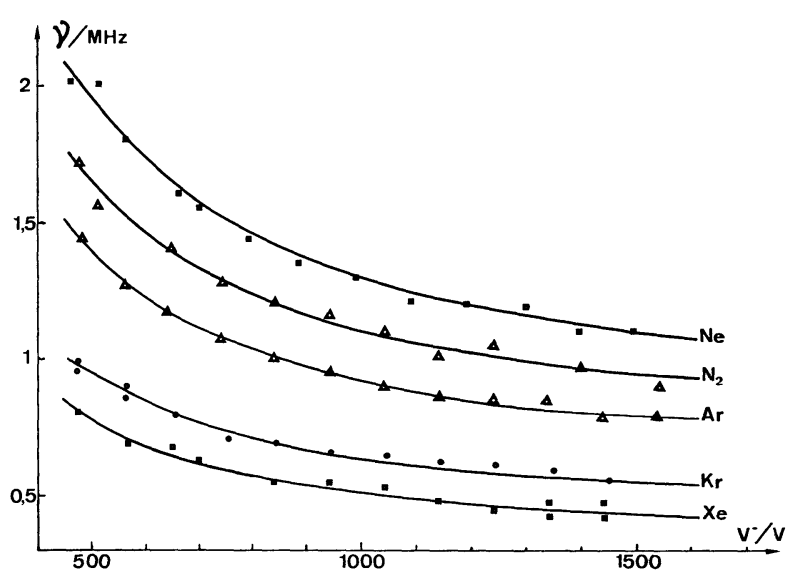

FIG. 9. - Influence de la tension d'accélération des électrons rapides sur la fréquence des oscillations $\left(I^{-}=12,4 \mathrm{~mA}\right.$, $\left.V_{\mathrm{ex}}^{+}=4000 \mathrm{~V}\right)$.

Là encore nous voyons que la fréquence expérimentale suit une loi de variation en fonction de $V^{-}$analogue à la relation (3.4) et que les valeurs sont sensiblement égales à celles prévues par cette relation.

Ce bon accord entre les résultats expérimentaux et la relation (3.4) semble bien confirmer que nous avons affaire à des oscillations ioniques de type plasma. Nous avons aussi vérifié que la fréquence est indépendante du champ magnétique régnant dans la chambre et qu'elle varie très peu avec la pression, ce qui ne nous permet pas d'attribuer ces oscillations aux types d'ondes fréquemment rencontrées dans des expériences du même type telles que les ondes de Alfven, les ondes cyclotronique ou magnétron.

5. Etude de la variation d'énergie des ions au moment de la discontinuité. - Les diverses discontinuités observées sur les courbes des figures 3 et 4 sont accompagnées d'une brusque variation de l'énergie des ions extraits. La tension $V_{\text {ex }}^{+}$appliquée à l'électrode d'extraction étant maintenue constante, nous devons admettre que c'est le potentiel au point où est créé l'ion qui varie. Si nous désignons par $\varphi_{\mathrm{e}}$ ce potentiel, tout se passe comme si les ions étaient extraits sous une d. d. p.

$$
V^{+}=V_{\mathrm{ex}}^{+}+\varphi_{\mathrm{e}} \text {. }
$$

L'induction magnétique régnant dans l'entrefer de l'électro-aimant d'analyse et qui permet d'obtenir une focalisation optimum du faisceau d'ions analysés est reliée à cette $\mathrm{d}$. $\mathrm{d}$. $\mathrm{p}$. et aux caractéristiques de l'ion analysé par la relation :

$$
R=\frac{1}{B}\left[\frac{2 m^{+}}{q}\left(V_{\mathrm{ex}}^{+}+\varphi_{\mathrm{e}}\right)\right]^{1 / 2}
$$

$R$ est le rayon du secteur magnétique $m^{+}$et $q$ sont respectivement la masse et la charge de l'ion considéré.

Si $\varphi_{\mathrm{e}}$ varie, pour obtenir à nouveau une focalisation optimum des ions analysés on doit modifier $B$ et on a sensiblement :

$$
\Delta \varphi_{\mathrm{e}}=2 \frac{\Delta B}{B} V_{\mathrm{ex}}^{+}
$$

La mesure de $\Delta B$ permet donc de déterminer la variation $\Delta \varphi_{\mathrm{e}}$ du potentiel au point où est créé l'ion. Sur la figure 10, nous avons représenté en fonction de $I^{-}$la variation d'énergie $\Delta \varphi_{\mathrm{e}}$ des ions se produisant au moment de la discontinuité $C$ de la courbe 1 de la figure 3. $\Delta \varphi_{\mathrm{e}}$ est une fonction linéaire de $I^{-}$.

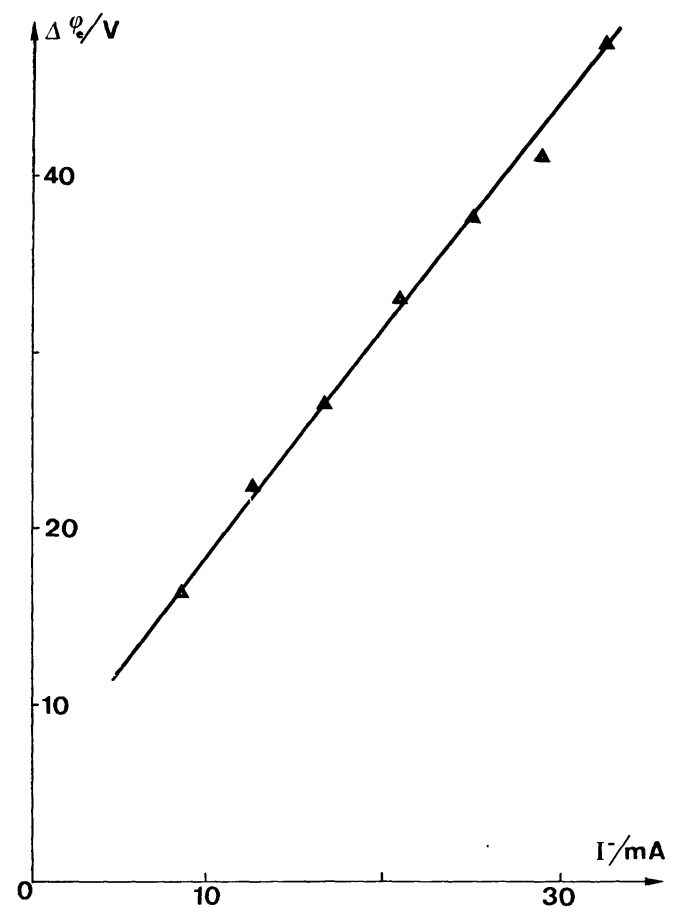

FIg. 10. - Etude de la variation d'énergie des ions au moment de la discontinuité en fonction de l'intensité du faisceau électronique (argon, $V^{-}=800 \mathrm{~V}, V_{\mathrm{ex}}^{+}=4000 \mathrm{~V}$ ).

Si nous admettons qu'en l'absence des oscillations le potentiel d'espace est sensiblement égal au potentiel de la chambre (potentiel 0$), \Delta \varphi_{\mathrm{e}}$ représente alors le potentiel $\varphi_{\mathrm{e}}$ au point où est créé l'ion lorsque la chambre est le siège des oscillations. Nous pouvons comparer cette valeur au potentiel $\varphi$ qui serait créé par le faisceau électronique seul en un point intérieur à ce faisceau, à une distance $r$ de l'axe soit :

$$
\varphi=\frac{3 \times 10^{4} I^{-}}{\sqrt{V^{-}}}\left[\log \frac{a}{r_{\mathrm{c}}}+\frac{r^{2}-a^{2}}{2 a^{2}}\right]
$$

$a$ et $r_{\mathrm{c}}$ étant respectivement le rayon moyen du faisceau et celui de la chambre.

Nous voyons que, bien que $\varphi_{\mathrm{e}}$ ne soit pas directement proportionnel à $I^{-}$, il semble logique de considérer que tout se passe comme si le faisceau n'était que par- 
tiellement neutralisé lorsque la chambre est le siège d'oscillations. S'il en est bien ainsi le potentiel en un point quelconque à l'intérieur de la chambre, à l'extérieur du faisceau doit aussi être différent de zéro.

L'utilisation d'une sonde de Langmuir est le moyen le plus simple à mettre en œuvre expérimentalement pour obtenir cette répartition. Cependant la présence du champ magnétique intense entraîne une modification des caractéristiques sonde, en particulier elles ne présentent aucun coude de saturation électronique. Dans ces conditions il est difficile de définir avec précision le potentiel d'espace. Tout ce que l'on peut évaluer c'est le potentiel flottant de la sonde, or cette évaluation peut être faussée par la présence des électrons rapides et par l'existence des oscillations [5]. Nous avons cependant mesuré le potentiel flottant d'une sonde introduite radialement par la tubulure $\mathrm{T}_{2}$. Sur la figure 11 la courbe 1 représente la variation de ce potentiel flottant à l'extérieur du faisceau en fonction de la distance $r$ de la sonde à l'axe du faisceau. Cette mesure est faite lorsque la chambre est le siège des oscillations étudiées ci-dessus. La courbe 2 , au contraire, représente la variation du potentiel flottant en fonction de $r$ en l'absence de ces oscillations.

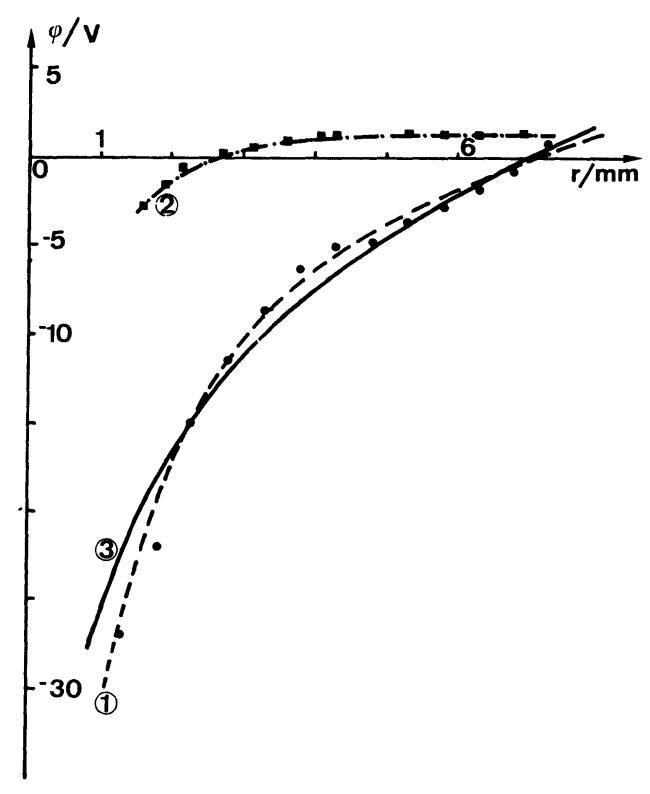

FIG. 11. - Etude־ de la répartition radiale de potentiel dans la chambre.

Malgré les restrictions précédentes, il semble logique d'affirmer, qu'en présence des oscillations, la répartition de potentiel dans la chambre n'est pas la même. En l'absence des oscillations, nous pouvons admettre que le volume de la chambre est sensiblement équipotentiel et que le milieu ionisé se comporte comme un plasma. En présence des oscillations la chambre présente un fort gradient radial de potentiel. La courbe 3 de la figure 11 représente, à titre indicatif, le potentiel qui serait créé par le faisceau d'électrons rapides, non neutralisé, en un point situé à l'extérieur du faisceau soit :

$$
\varphi=\frac{3 \times 10^{4} I^{-}}{\sqrt{V^{-}}} \log \frac{r}{r_{\mathrm{c}}}
$$

$r$ représente la distance du point à l'axe du faisceau, $r_{\mathrm{c}}$ est le rayon de la chambre.

Dans l'expérience dont les résultats sont reportés sur la figure $11 I^{-}=12,4 \mathrm{~mA}, V^{-}=800 \mathrm{~V}$.

Il semble donc logique de considérer qu'en présence des oscillations, le faisceau électronique n'est que partiellement neutralisé. Nous avons admis en outre $\rho^{+}=-\rho^{-}$, cette hypothèse étant vérifiée, d'une part par le fait qu'aux basses pressions il n'est pas possible d'obtenir des oscillations synchrones et, d'autre part, par l'accord entre les valeurs expérimentales de la fréquence et celles prévues par la relation (3.4). Pour concilier cette hypothèse avec les observations expérimentales, nous devons admettre aussi la présence d'électrons lents, provenant de l'ionisation, dans le faisceau d'électrons rapides. Or, dans tout ce qui précède nous n'avons pas tenu compte de la présence de ces électrons lents. Ces électrons peuvent intervenir de plusieurs manières : soit par leur rôle d'écran, soit par le champ électrique qu'ils créent. Nous pensons, comme Kino [6], que la faible mobilité radiale des électrons lents due à la présence du champ magnétique intense les empêche de jouer leur rôle d'écran vis-à-vis des ions dans leur mouvement transversal. Ils contribuent comme les électrons rapides à créer un champ radial qui se traduit par la répartition de potentiel radiale que nous venons de voir. Nous pensons que cette répartition est nécessaire à l'entretien du mouvement. Si le faisceau était neutralisé, il ne pourrait pas y avoir entretien de telles oscillations sans apport d'énergie extérieure. Ces électrons lents qui ne peuvent pratiquement s'écouler qu'axialement se trouvent piégés, à droite, par le potentiel d'extraction des ions, à gauche, par le potentiel de charge d'espace négatif créé, à l'extérieur de la chambre, par le faisceau d'électrons rapides. Dans cette région le faisceau électronique est encore très concentré à cause du champ magnétique y régnant, par contre la pression est faible par rapport à celle existant dans la chambre. Le faisceau électronique est donc peu neutralisé créant ainsi un potentiel de charge d'espace négatif important. Cette double hypothèse se trouve vérifiée par les deux observations suivantes : d'abord si nous réalisons des expériences uniquement avec le faisceau incident, c'est-à-dire que nous n'appliquons aucune tension d'extraction des ions, il n'est pas possible de mettre en évidence les oscillations précédentes. Dans ce cas les électrons lents peuvent s'écouler axialement vers la droite et le faisceau tend à se neutraliser progressivement. Nous mettons en évidence le rôle joué par la partie du faisceau située à gauche de la chambre en faisant croître la pression dans cette région, comprise entre la chambre et le canon à électrons en diminuant la vitesse de pompage dans l'enceinte à laquelle est reliée cette partie, la 
pression dans la chambre n'est pas modifiée, nous ne pouvons plus mettre en évidence l'apparition d'oscillations cohérentes. Lorsque nous faisons croître la pression dans cette région située à l'extérieur de la chambre, le nombre d'ions créés y augmente, le faisceau électronique tend à s'y neutraliser plus rapidement ne créant plus une barrière de potentiel s'opposant au départ des électrons lents de la chambre. Le faisceau tend alors à se neutraliser dans cette dernière, il ne peut pas y avoir entretien des oscillations.

6. Conclusion. - La source d'ions que nous avons mise au point, bien que de performances modestes, nous semble être un outil de travail intéressant en vue de l'étude fondamentale des sources à confinement magnétique. Les principaux paramètres sont parfaitement indépendants les uns des autres et mesurables avec une bonne précision.
Nous avons mis en évidence le rôle primordial joué par des oscillations transversales, de type plasma, des ions. Ces oscillations sont excitées par le mouvement individuel des ions dans le puits de potentiel radial créé par le faisceau d'électrons rapides. Les discontinuités dans le courant ionique extrait sont dues à l'apparition ou à la disparition de ces oscillations.

Remerciements. - Je remercie M. le Professeur Vauthier Directeur du Laboratoire de Spectrométrie de masse de la Faculté des Sciences de Poitiers qui m'a suggéré ce travail et qui m'a guidé au cours de son évolution.

Je voudrais aussi exprimer toute ma gratitude à M. le Professeur Gautherin qui a suivi le développement de cette étude, ses conseils, les idées extrêmement fructueuses qu'il m'a suggérées m'ont permis de la mener à bien.

\section{Bibliographie}

[1] Gautherin, G., Thèse d'Etat, Orsay 1967, p. 29.

[2] Chantreau, J., Thèse d'Etat, Poitiers 1970, p. 38.

[3] Hernquist, K. G., J. Appl. Phys. 26 (1955) 544 à 548.

[4] Machet, J, Thèse d'Etat, Poitiers 1974, p. 61.
[5] Kagan, Yu. M., Perel, V. I., Sov. Phys. Usp. (1964) 767 à 793.

[6] KINo, Proceedings of the Sixth International Conference on Ionization Phenomena in gases (Paris) 3 (1963) 219-221. 